\title{
New Action Principle for Classical Particle Trajectories in Spaces with Torsion
}

\author{
P. Fiziev* and H. Kleinert \\ Institut für Theoretische Physik, \\ Freie Universität Berlin \\ Arnimallee 14, D - 14195 Berlin
}

(March 24, 2018)

\begin{abstract}
To comply with recent developments of path integrals in spaces with curvature and torsion we find the correct variational principle for the classical trajectories. Although the action depends only on the length, the trajectories are autoparallels rather than geodesics due to the effects of a new torsion force.
\end{abstract}

03.20. $+\mathrm{i}$

04.20.Fy

$02.40 .+\mathrm{m}$

Typeset using REVTEX

*Permanent address: Department of Theoretical Physics, Faculty of Physics, University of Sofia, Bull. 5 James Boucher, Sofia 1126, Bulgaria. Work supported by the Commission of the European Communities for Cooperation in Science and Technology, Contract No. ERB3510PL920264 
1) Recently it was shown [1,2] that in order to obtain a consistent path integral for point particles moving in a space with curvature and torsion, the classical trajectories must necessarily be autoparallels rather than geodesics. Whereas geodesics are described by

$$
\ddot{q}^{\nu}+\bar{\Gamma}_{\alpha \beta}{ }^{\nu} \dot{q}^{\alpha} \dot{q}^{\beta}=0,
$$

with Christoffel symbols $\bar{\Gamma}_{\mu \nu}{ }^{\lambda}$, the equations of motion of autoparallels are

$$
\ddot{q}^{\nu}+\Gamma_{\alpha \beta}{ }^{\nu} \dot{q}^{\alpha} \dot{q}^{\beta}=0,
$$

where $\Gamma_{\mu \nu}{ }^{\lambda}$ are the affine connections (related to the Christoffel via $\Gamma_{\mu \nu \lambda}=\bar{\Gamma}_{\mu \nu \lambda}+S_{\mu \nu \lambda}-$ $S_{\nu \lambda \mu}+S_{\lambda \mu \nu}$ where $S_{\mu \nu \lambda}$ is the torsion tensor).

This problem is quite general and appears in different branches of physics. For example, in the past, the general theory of relativity has been extended to spaces with torsion [3]. Assuming plausible actions and the usual field theoretic formalism it has been argued that the orbits of spinless particles are geodesics. Since up to now no possibility seems to exist to falsify this result experimentally, the authors have been on safe although rather academic grounds. This situation has been changed drastically with the findings in the context of path integrals in Ref. [2]. It has been known for a long time that the equations of motion of nonrelativistic celestial mechanics can be solved most efficiently by subjecting them to a Kustaanheimo-Stiefel transformation [ [i]. It has also been known that when transforming the action likewise, its variation does not yield the correct equations of motion. The reason lies in the nonholonomic nature of the transformation. This transformation has been shown [2] to introduce torsion into the new coordinate system. Thus, the description of celestial bodies in these coordinates can serve as an ideal experimentally well-known testing ground for any theory in spaces with torsion. In Ref. [2] it was shown that the trajectories of celestial bodies in this space move along the autoparallels rather than the geodesics. Although at first sight unrelated, this undisputable fact calls for a revision of a large body of theory developed in the context of general relativity in spaces with torsion.

The fact that the trajectories are autoparallels agrees with Einstein's equivalence principle according to which the correct dynamical equations in a noneuclidean space may be 
found by transforming the free-particle equations of motion in a flat space $\ddot{x}^{i}(t)=0$ locally to arbitrary curvilinear coordinates via some differential transformation

$$
d x^{i}=e_{\mu}^{i}(q) d q^{\mu}
$$

The transformed equations are postulated to describe the orbits $q^{\mu}(t)$ in the curved space with the metric

$$
g_{\mu \nu}(q)=e_{\mu}^{i}(q) e_{\nu}^{i}(q)
$$

In the presence of torsion, the transformation (3) carries the equations of motion into (2), not (11).

The key to the present work lies in a problem which arises when trying to apply Einstein's equivalence principle to the action of the free particle

$$
\mathcal{A}=\int_{t_{a}}^{t_{b}} d t \frac{M}{2} \dot{x}^{2}(t)
$$

Under the transformation (3), this goes over into

$$
\mathcal{A}=\int_{t_{a}}^{t_{b}} d t \frac{M}{2} g_{\mu \nu}(q(t)) \dot{q}^{\mu}(t) \dot{q}^{\nu}(t)
$$

If extremized in the standard way [5], it would yield the euqations of motion (1), i.e., the the wrong trajectories in the presence of torsion.

The purpose of this note is to resolve this conflict by exhibiting a new variational principle in spaces with torsion where action (6) yields autoparallels after all.

2) The important point to realize is that in order to obtain the correct trajectories from

\footnotetext{
${ }^{1}$ The new action principle we propose here has different physical applications. In this article we ilustrate the main idea of this principle only on the simplest example of nonrelativistic particle in tree dimensional space. Therefore we use here an euclidean metric in the x-space, which is enough for nonrelativistic problems. To get the relastvistic signature of the metric in the corresponding four-dimensional space with torsion one has to put a Minkovski metric into a flat four-dimensional x-space.
} 
the transformed action (6), the variations $\delta q^{\mu}(t)$ have to be performed in accordance with independent variations $\delta x^{i}(t)$ in the flat space which vanish at the endpoints (see Fig. 1a). The paths in the two spaces are related by the integral equation

$$
q^{\mu}(t)=q^{\mu}\left(t_{a}\right)+\int_{t_{a}}^{t} e_{i}^{\mu}\left(q\left(t^{\prime}\right)\right) \dot{x}^{i}\left(t^{\prime}\right) d t^{\prime}
$$

where $e_{i}^{\mu}(q)$ is the inverse matrix of $e_{\mu}^{i}(q)$ in (3). It is easy to derive from this an explicit, albeit nonlocal, equation between $\delta x^{i}(t)$ and $\delta q^{\mu}(t)$. For this we introduce the auxiliary quantities

$$
\delta_{h} q^{\mu} \equiv e_{i}{ }^{\mu}(q) \delta x^{i}
$$

which are equivalent to the independent variations $\delta x^{i}(t)$ to be performed on the system. From (3) we find the relation

$$
\delta \dot{q}^{\mu}(t)=\frac{d}{d t} \delta_{h} q^{\mu}+2 \dot{q}^{\nu} S_{\nu \lambda}^{\mu} \delta_{n} q^{\lambda}-\delta \Delta^{\lambda} \Gamma_{\lambda \nu}{ }^{\mu} \dot{q}^{\nu}
$$

where

$$
\delta \Delta^{\mu} \equiv \delta q^{\mu}-\delta_{h} q^{\mu}
$$

Using (7) we see that

$$
\delta \dot{q}^{\mu}(t)=\frac{d}{d t} \delta q^{\mu}(t)
$$

so that (9) can be rewritten as

$$
\frac{d}{d t} \delta \Delta=-\delta \Delta^{\lambda} \Gamma_{\lambda \nu}^{\mu} \dot{q}^{\nu}+2 \dot{q}^{\nu} S_{\nu \lambda}^{\mu} \delta_{h} q^{\lambda}
$$

This differential equation can be integrated to

$$
\delta \Delta(t)=\int_{t_{a}}^{t} d t^{\prime} U_{t t^{\prime}} \quad \sigma\left(t^{\prime}\right)
$$

where

$$
U_{t t^{\prime}}=T \exp \left[-\int_{t^{\prime}}^{t} G\left(t^{\prime \prime}\right) d t^{\prime \prime}\right]
$$


We have used vector and matrix notation with

$$
\begin{aligned}
G_{\mu}{ }^{\lambda}(t) & =\Gamma_{\mu \nu}^{\lambda}(q(t)) \dot{q}^{\nu}(t) \\
\sigma^{\mu}(t) & =2 \dot{q}^{\nu}(t) S_{\nu \lambda}{ }^{\mu}(q(t)) \delta_{h} q^{\lambda}(t) .
\end{aligned}
$$

If the space is free of torsion, then $\sigma(t) \equiv 0$ and hence $\delta \Delta(t) \equiv 0$. In this case the variations $\delta q^{\mu}(t)$ coincide with the independent ones $\delta_{h} q^{\mu}(t)$. We shall therefore refer to $\delta_{h} q^{\mu}(t)$ as the holonomic variations of the path $q^{\mu}(t)$. In the presence of torsion, on the other hand, the variations $\delta q^{\mu}(t)$ differ from $\delta_{h} q^{\mu}(t)$. We shall emphasize the nonholonomic properties of $\delta q^{\mu}$ by writing them as $\delta q^{\mu}$, in analogy with the notation $\mathrm{d} Q$ used in thermodynamics for a small increment of a nonintegrable function.

Let us calculate the variation of the action which is

$$
\delta A=M \int_{t_{a}}^{t_{b}} d t\left(g_{\mu \nu} \dot{q}^{\nu} \delta \dot{q}^{\mu}+\frac{1}{2} \delta q^{\mu} \partial_{\mu} g_{\alpha \beta} \dot{q}^{\alpha} \dot{q}^{\beta}\right)
$$

The most direct way of deriving from $\delta A$ the equations of motion is by inserting an infinitesimal local "knock" variation

$$
\delta_{h} q^{\mu}(t)=\epsilon^{\mu} \delta\left(t-t_{0}\right)
$$

In the torsion-free case with $\delta q^{\mu}(t) \equiv \delta_{h} q^{\mu}(t)$ we obtain

$$
\delta A \equiv \delta_{h} A=-\epsilon^{\mu} M g_{\mu \nu}\left(\ddot{q}^{\nu}+\bar{\Gamma}_{\alpha \beta}^{\nu} \dot{q}^{\alpha} \dot{q}^{\beta}\right)
$$

Setting this equal to zero produces at once the equations of motion which are those of geodesics, i.e., the correct particle trajectories in the absence of torsion.

In the presence of torsion, the holonomic "knock" variation (18) leads via (13) - (16) to the total variation

$$
\delta q(t)=\epsilon \delta\left(t-t_{0}\right)+\delta \Delta(t)
$$

with

$$
\delta \Delta(t)=\Theta\left(t-t_{0}\right) U_{t t_{0}} \sigma\left(t_{0}\right)
$$


Note that the term $\delta \Delta(t)$ is nonlocal. It has the important effect that while $\delta q^{\mu}\left(t_{a}\right)=0$, the variation at the endpoint $\delta q^{\mu}\left(t_{b}\right)=b^{\mu}$ is nonzero. The vector $b^{\mu}$ corresponds to the Burgers vector in the physics of dislocations [4]. The situation is illustrated in Fig. 1c.

The nonlocal term modifies the usual derivation of the equations of motion. The time derivative of $\delta \dot{q}(t)$ is

$$
\delta \dot{q}(t)=\epsilon \dot{\delta}\left(t-t_{0}\right)+\delta\left(t-t_{0}\right) \Sigma\left(t_{0}\right) \epsilon-G \delta \Delta(t)
$$

where $\Sigma_{\mu}^{\lambda}(t)=2 S_{\mu \nu}{ }^{\lambda}\left(q(t) \dot{q}^{\nu}(t)\right.$. Inserting $\delta q^{\mu}(t)$ and $\delta \dot{q}^{\mu}(t)$ into (17) we obtain $\delta A=$ $\delta_{h} A+\delta_{a} A$, with the anholonomic deviation $\delta_{a} A$ of the action

$$
\delta_{a} A=-\epsilon^{\mu} 2 M S_{\mu \alpha \beta}\left(q\left(t_{0}\right)\right) \dot{q}^{\alpha}\left(t_{0}\right) \dot{q}^{\beta}\left(t_{0}\right)
$$

originating from the second term in (22). The contribution of $-G \delta \Delta(t)$ in (22) cancels exactly the contribution of the term $\delta \Delta^{\mu} \frac{1}{2} \partial_{\mu} g_{\alpha \beta} \dot{q}^{\alpha} \dot{q}^{\beta}$ which originates from the second term in $\delta A(17)$, because of (15) and the identity $\frac{1}{2} \partial_{\mu} g_{\alpha \beta}-\Gamma_{\mu\{\alpha \beta\}} \equiv 0$ which follows directly from the definitions $g_{\alpha \beta}=e^{i}{ }_{\alpha} e^{i}{ }_{\beta}$ and $\Gamma_{\mu \nu}{ }^{\lambda}=e_{i}{ }^{\lambda} \partial_{\mu} e^{i}{ }_{\nu}$ (it expresses the fact that the covariant derivative of the metric tensor vanishes, $D_{\mu} g_{\alpha \beta} \equiv 0$.).

Hence the total variation of the action is

$$
\begin{aligned}
\delta A & =-M \epsilon^{\mu} g_{\mu \nu}\left[\ddot{q}^{\nu}+\left(\bar{\Gamma}_{\alpha \beta}^{\nu}-2 S_{\alpha \beta}^{\nu}\right) \dot{q}^{\alpha} \dot{q}^{\beta}\right] \\
& =-M \epsilon^{\mu} g_{\mu n}\left(\ddot{q}^{\nu}+\Gamma_{\alpha \beta}^{\nu} \dot{q}^{\alpha} \dot{q}^{\beta}\right)
\end{aligned}
$$

the second line following from the first via the identity $\bar{\Gamma}_{\alpha \beta}{ }^{\nu}=\Gamma_{\{\alpha \beta\}}{ }^{\nu}+2 S_{\{\alpha \beta\}}^{\nu}$. Setting $\delta A=0$ gives the autoparallels as the equations of motions which is what we wanted to demonstrate. 


\section{REFERENCES}

[1] H. Kleinert, Mod. Phys. Lett. A 4, 2329 (1989)

[2] H. Kleinert, Path Integrals in Quantum Mechanics, Statistics and Polymer Physics, World Scientific Publishing Co., Singapore 1990. Second extended edition in German publ. by B.I.-Wissenschaftsverlag, Mannheim, 1993.

[3] See, for instance, the review by

F.W. Hehl, P. v.d. Heyde, G.D. Kerlick, and J.M. Nester, Rev. Mod. Phys. 48, 393 (1976).

[4] P. Kustaanheimo and E. Stiefel, J. Reine Angew. Math. 218, 204 (1965).

See also the textbook

E. Stiefel and G. Scheifele, Linear and Regular Celestial Mechanics, Springer, Berlin, 1971.

[5] V.I. Arnold, Mathematical Methods of Classical Mechanics, Springer Verlag, New York Heidelberg Berlin, 1984.

[6] B.A. Billy, R. Bullough, and E. Smith, Proc. Roy. Soc. A231 (1955) 263;

F. Kroupa and P.B. Price, Phil. Mag. 6 (1961) 243;

H. Kleinert, Gauge Fields in Condensed Matter, Vol. II Stresses and Defects World Scientific Publishing Co., Singapore 1989. 
FIGURE 1 In the usual holonomic case, the paths $x(t)$ and $x(t)+\delta x(t)$ shown in (a) are mapped into paths $q(t)$ and $q(t)+\delta_{h} q(t)$ shown in (b). In the nonholonomic case with $S_{\alpha \beta}^{\nu} \neq 0$, however, they go over into $q(t)$ and $q(t)+\delta q(t)$ shown in (b) with a failure to close at $t_{b}$ by a vector $\mathbf{b}$, the analog to the Burgers vector in a solid with dislocations. 